FDL TDR 64-91

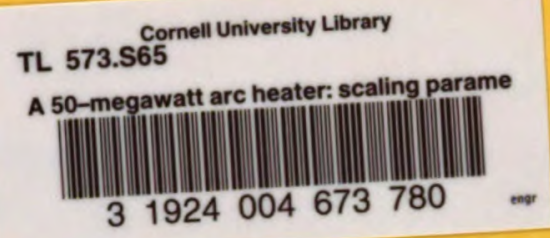

\title{
A 50-MEGAWATT ARC HEATER: SCALING PARAMETERS AND PERFORMANCE PREDICTION
}

FICHARD T. SMITH

JOSEPH P. DOYLE

TECHNICAL DOCUMENTARY REPORT No. FDL TDR 64-91

AUGUST 1964

\author{
AF FLIGHT DYNAMICS LABORATORY \\ /RESEARCH AND TECHNOLOGY DIVISION ) \\ AIR FORCE,SYSTEMS COMMAND \\ WRIGHT-PATTERSON AIR FORCE BASE, OHIO
}

Project No. 1426, Task No. 142612 